Journal of Engineering and Applied Sciences 14 (8): 2409-2417, 2019

ISSN: 1816-949X

(C) Medwell Journals, 2019

\title{
A Study on the Crying Characteristics of Carnivores and Herbivores using Acoustic Signal Analysis
}

\author{
${ }^{1} \mathrm{Ik}$-Soo Ahn, ${ }^{1}$ Myung-Jin Bae and ${ }^{2}$ Seong-Geon Bae \\ ${ }^{1}$ Department of Information and Telecommunication, Soongsil University, \\ 369 Sangdo-ro Dongjak-gu, Seoul, Korea \\ ${ }^{2}$ School of Software Application, Kangnam University, Gyunggi-do, Korea
}

\begin{abstract}
Global warming, El Nino and various environmental disasters have made the natural wildlife protection area disappear. Animals in the wild form a moderate food chain relationship but recently the food chain has been disrupted. The phenomenon can be seen from the frequency of occurrence of carnivorous crying and the incidence of herbivorous crying in African grasslands. If so, it is necessary to try to restore the food chain system of safari if artificial manipulation of the cry of predator and herbivorous animals by the reverse idea. The research method is similar to the crying of elephants, zebras and deer which are representative animals of tiger, lion, puma and herbivorous animals which are representative animals of wild animals among wild animals. Respectively in order to compare the characteristics of the crying of carnivorous and herbivorous animals, we compared the cry of the elephant among tiger and herbivorous animals. The purpose of this study is to contribute to the creation and restoration of ecological environment for the wild animals and to contribute to the rearing of the animals in the zoos by letting the other person hear the cries of the mutually related animals.
\end{abstract}

Key words: Food chain, carnivorous, herbivorous, crying, natural enemies, ecological environment, zoo, health care

\section{INTRODUCTION}

Animals have their own unique cries. Animals do not develop language like humans but they use crying to communicate at least with each other. An animal's crying can also be used to threaten the other, to use it as a defense to protect oneself from other threats or to find a mating partner. Among them, carnivorous animals and herbivores are eaten and eaten together, so, crying is also, a crying cry that threatens to survive or is used as a defense. Carnivorous animals threaten herbivores when she hunts and make a crying whine. Herbivores live mainly in flocks, so, they give a defensive cry to the predator, the carnivore or to warn their coworkers. From that point of view, the cries of carnivores and herbivores have different characteristics. In this study, we compare and analyze the characteristics of the carnivorous crying and herbivorous crying and investigate the reason for such crying (Sung-Hoon and Myung-Jin, 2007; Chan-Jung and Myung-Jin, 2007; Hoon and Jin, 2010; Poole et al., 2005).

\section{MATERIALS AND METHODS}

Outline of electric power simple transportation device: Animals can be divided into carnivorous animals, herbivores and omnivorous animals based on their food intake. Many omnivorous animals are mainly domesticated animals. Wild animals are mostly carnivorous or herbivorous animals. A predator refers to an animal that feeds on animals while an herbivorous animal refers to an animal that eats grass or nuts. Its characteristics are relatively clear, depending on the subject of the prey. Predators are harsh, oppressive and cruel because they have to eat and eat live animals. Herbivorous animals are temperate and relaxed because they do not need much effort to obtain food (Poole et al., 2005; Beckers et al., 2004; Doo-Hoon and Myung-Jin, 2013; Se-Bin, 2014; Bum et al., 2012). A predator refers to a feline animal that eats animals, typically tigers, lions and cougars. Carnivores have to eat tough meat, so, their canines are large and sharp and their jaw muscles are strong. The tongue is also, well developed with roughness and elasticity because it needs to apply the hair and bones of the food well. Digestive organ is relatively simple compared to herbivore has a short

Corresponding Author: Myung-Jin Bae Department of Information and Telecommunication, Soongsil University, 
organs on top of it and enzymes that break down proteins break down and break down the meat. Also because of the need to hunt, all muscles of the body are well developed and agile and the claws are very sharp and strong. These oral structures and body structures such as digestive organs, affect the crying. The herbivorous animals mainly live in flocks and are animals that eat mainly plants such as leaves, fruits, fruits and grasses. Representative herbivorous mammals include small animals such as squirrels and rabbits, large animals such as elk, deer, cow and horse, giraffe and elephant. The plants consumed by the herbivorous animals are rich in fiber, so, they are tanned like crumbs and they are crushed and crushed. The digestive tract also has a structure for digesting fibrin and a lot of digestive juices are also, secreted. In particular, three deer, cattle, sheep, giraffe, etc. are four chambers in the stomach, several times to ruminate and digest. The horses have one stomach but digestive organs are developed in the large intestine, so that, they can ferment and digest for 2 days. These body structures such as oral structures and digestive organs, affect the crying.

\section{RESULTS AND DISCUSSION}

A comparison of the cry of carnivores and herbivores: The cries of carnivores and herbivores have unique characteristics. Carnivorous animals and herbivores are not only different in their eating habits but also in physical conditions and life styles. The carnivore approaches the living prey in an agile manner and moves rapidly and quickly rushes out and breaks the breath with a strong mouth and pushes over with all his body. It should also be eaten quickly to avoid being caught by other animals or before feeding. To adapt to these lifestyles, the body should not be too big, the skeleton must be strong, the whole body must be muscular, the claws and teeth must be sharp and the jaws must be strongly developed. Herbivorous animals are grass or fields, grassy or leaf-eating animals in the forest and are easily slim or very tiny animals to avoid large animals and predators. Most of them are living in groups because they have to keep each other out of fear that predators will always appear if they have in common. In addition, since, the food has a lot of fiber, it is grinded rather than the canine and grinds and digests for a long time, so that, the oral structure and the digestive organs are common only to carnivores and other herbivores. These body structures and activities suggest that carnivorous animals and herbivores have characteristics of crying that are opposed to each other. In order to compare the characteristics of the crying of carnivorous animals and herbivorous animals, we compared and analyzed the cry of three representative animals of carnivorous animals and three representative animals of herbivorous animals (Chan-Jung and Myung-Jin, 2007). Carnivores are predominantly feline animals, producing strong, low-frequency sounds with long, large trunks, thick necks, large and wide tongues, broad saints and sturdy vocal cords, predominantly rigid and flexible muscles and robust and large-volume lungs. These body structures and characteristics complement each other to produce a cry for the charisma of carnivores. In particular, carnivorous animals can produce a distinctive roaring sound because they have a resilient cartilaginous hyoid bone. I compared the acoustics of the carnivorous tigers, lions and puma.

Figure 1 compares the cry of the carnivorous tigers, lions and puma by time domain graph, spectrogram graph and frequency spectrum graph. As shown in the figure, the sound of carnivorous crying is that the resonance characteristics of thick and strong saints and the vibrating sound of thick and rough vocal cords are mutually intertwined by the large and flexible mouth structure and tongue, creating unique peculiar sounds of carnivores have. It can be seen that the cry of the carnivores emits a strong energy as well as a low frequency sound in the human audible frequency band as well as an ultra-low frequency sound outside the audible frequency band.

Also, the time domain graphs of a-c show a strong cry and long longevity. The spectrograms of a-c show that the strong energy is concentrated in the low frequency band. Especially in the spectrum comparison chart of $\mathrm{d}$, all three animal's exhibit strong frequency energy in the low frequency band of $1,000 \mathrm{~Hz}$ or less and show characteristic characteristics of carnivorous cry. In short, the cry of the carnivore is a sound with strong lowfrequency sound, strong and long lengthening sound and shaking the surface of the ground which shows that the whole body of the herbivorous animal is thrilled and has a sound characteristic (Hoon and Jin, 2010; Lee et al., 2008; Moon and Dae, 2015; Kyoung and Jin, 2015; SangHwi et al., 2017; Bae et al., 2013; Bae and Kim, 2013).

Herbivores are more diverse than predators with large elephants, horses, deer and deer in their normal size and small animals such as rabbits. Large animals such as elephants, use predominantly large masses to drive out predators while horses and deer are quick and run fast using their fast legs to avoid predators. These herbivores are always tense from the threats of predators and must cope with them. Herbivorous animals that are predominantly alert to predators will alert the crowd of the dangerous cries and generate distinctive, distinctive, high-frequency sounds. The elephant also uses ultra-low frequency sounds to find distant mates, so, it has a variety of timbres ranging from ultra-low frequency sounds to high frequency sounds. Horses and deer also, use both low and high frequency sounds (Poole et al., 2005). 
(a)
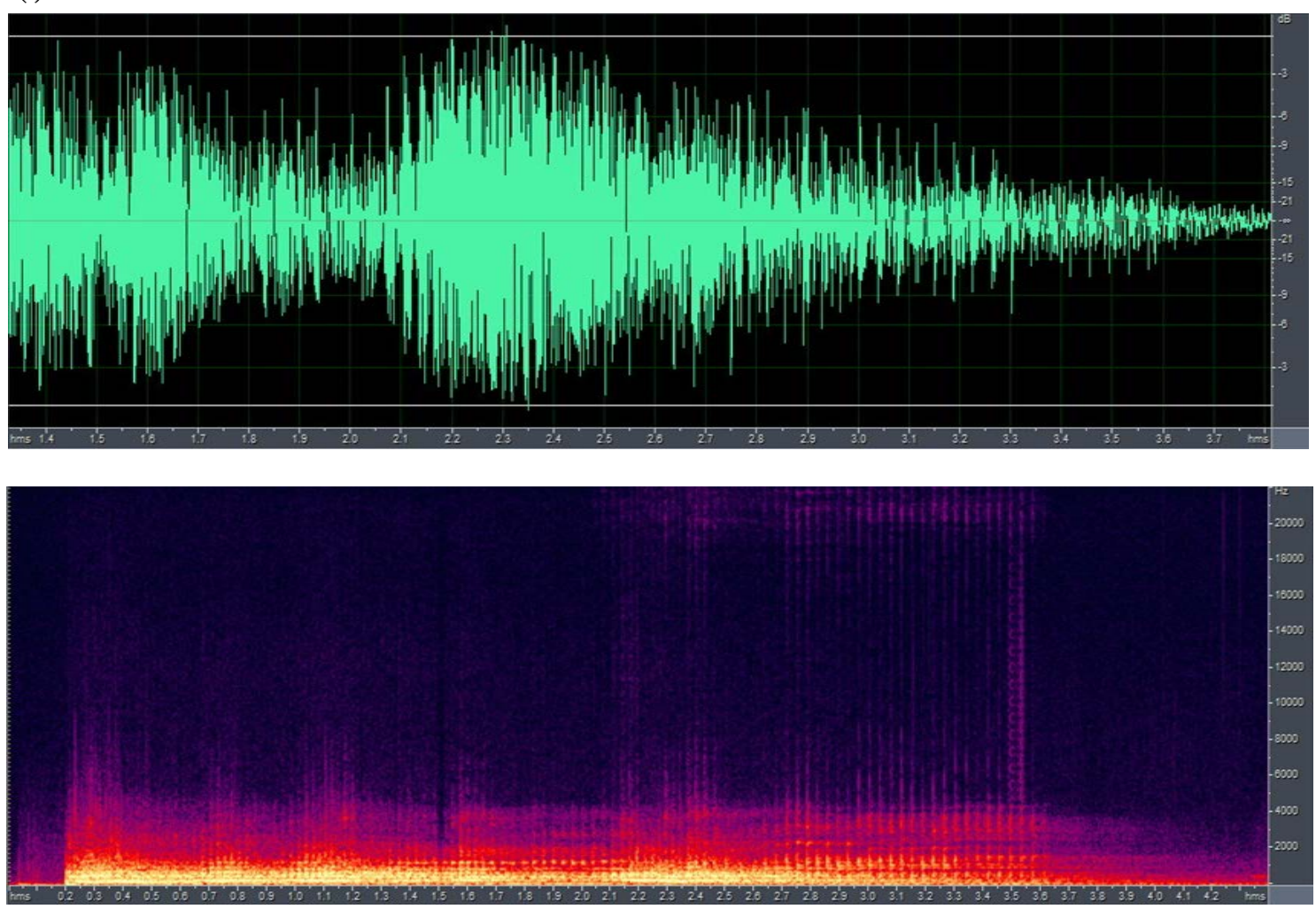

(b)
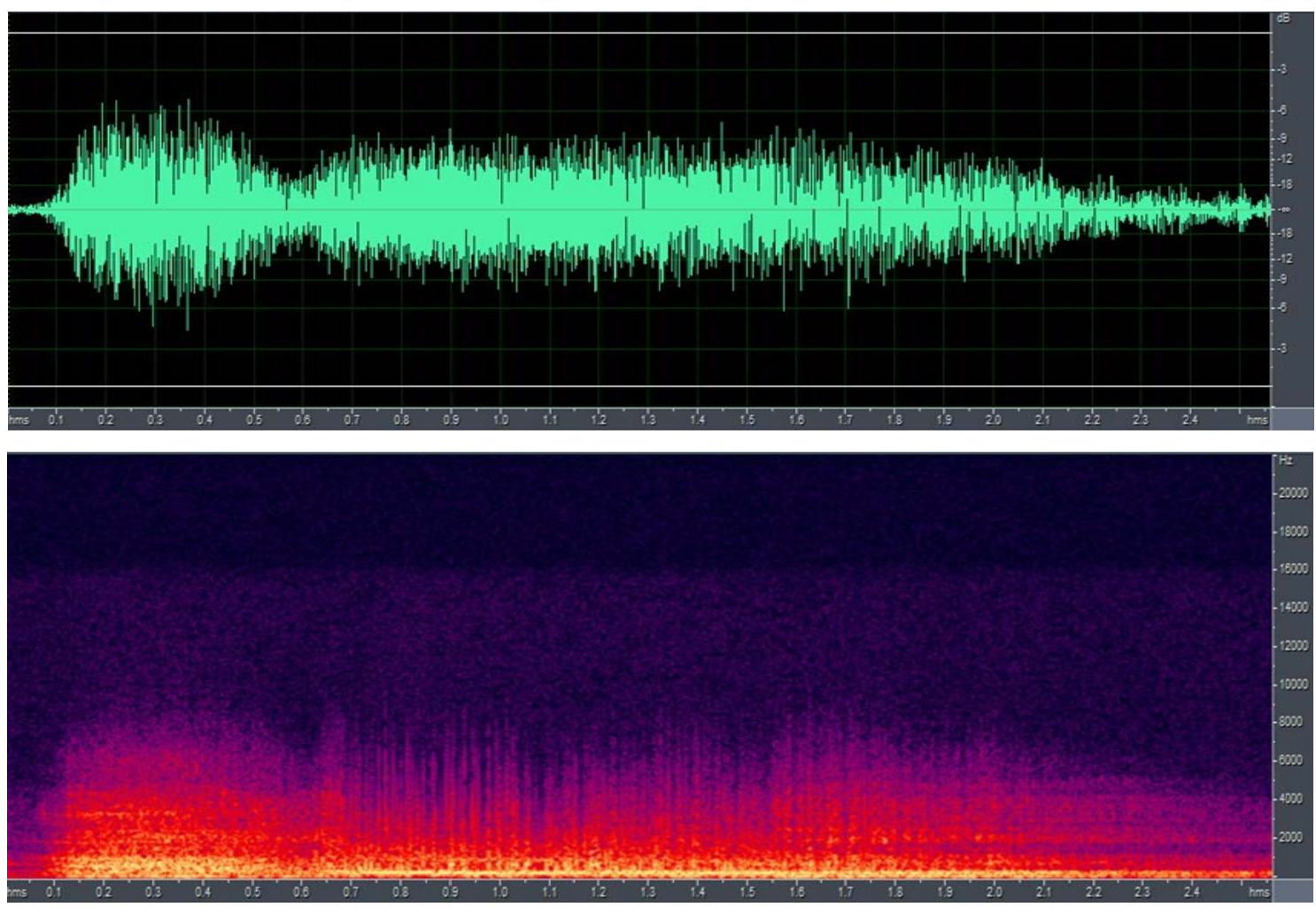

Fig. 1: Continue 



(d)

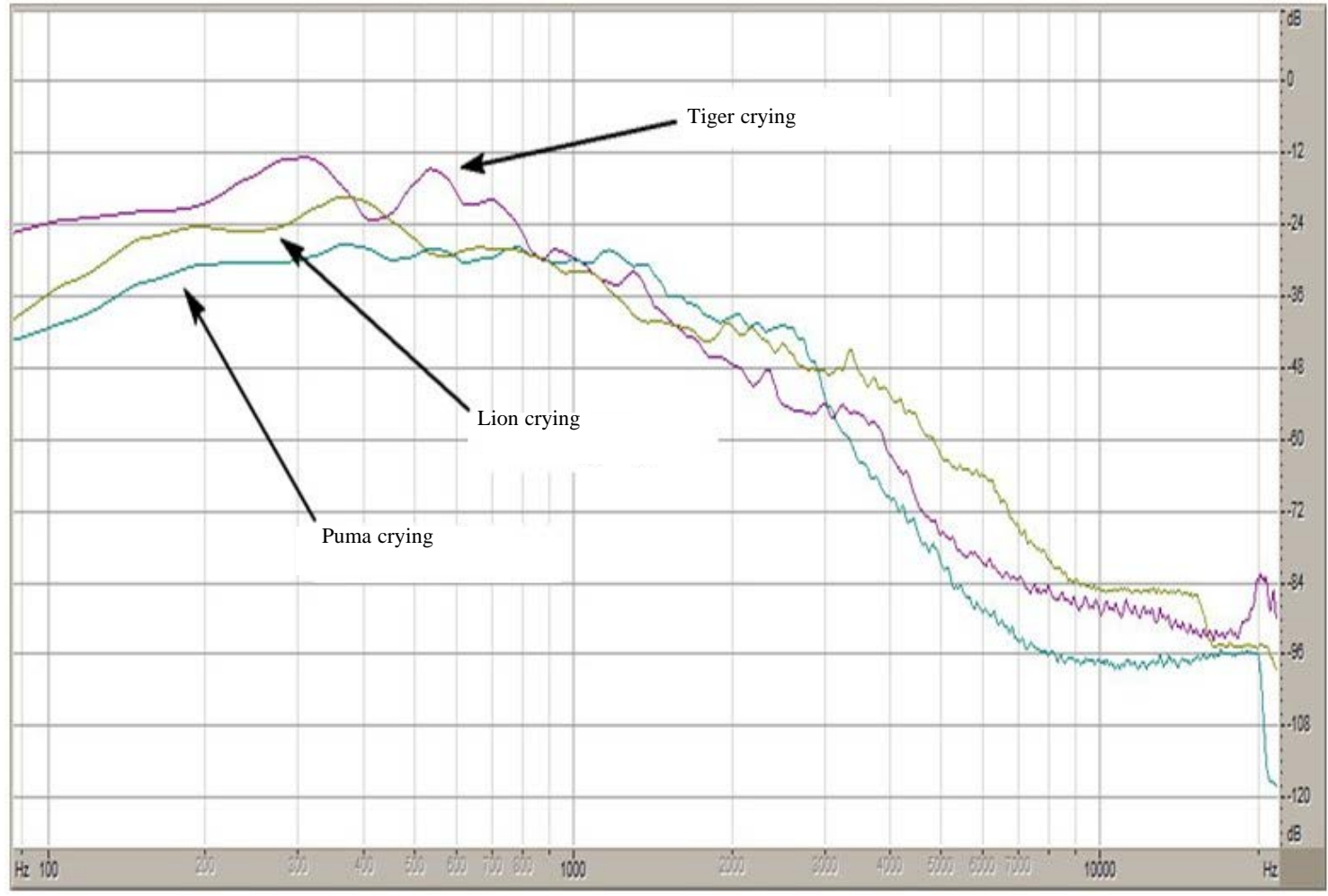

Fig. 1: a) Tiger crying; b) Lion crying; c) Puma crying and d) Carnivorous crying characteristics 


\section{J. Eng. Applied Sci., 14 (8): 2409-2417, 2019}

As shown in Fig. 2 when the herbivorous cryos are large, the low frequency band is strong and the low frequency band is weak. Instead, the mid-frequency bands which are in the $1,000-5,000 \mathrm{~Hz}$ band have a

(a)


Fig. 2: Continue 
(c)
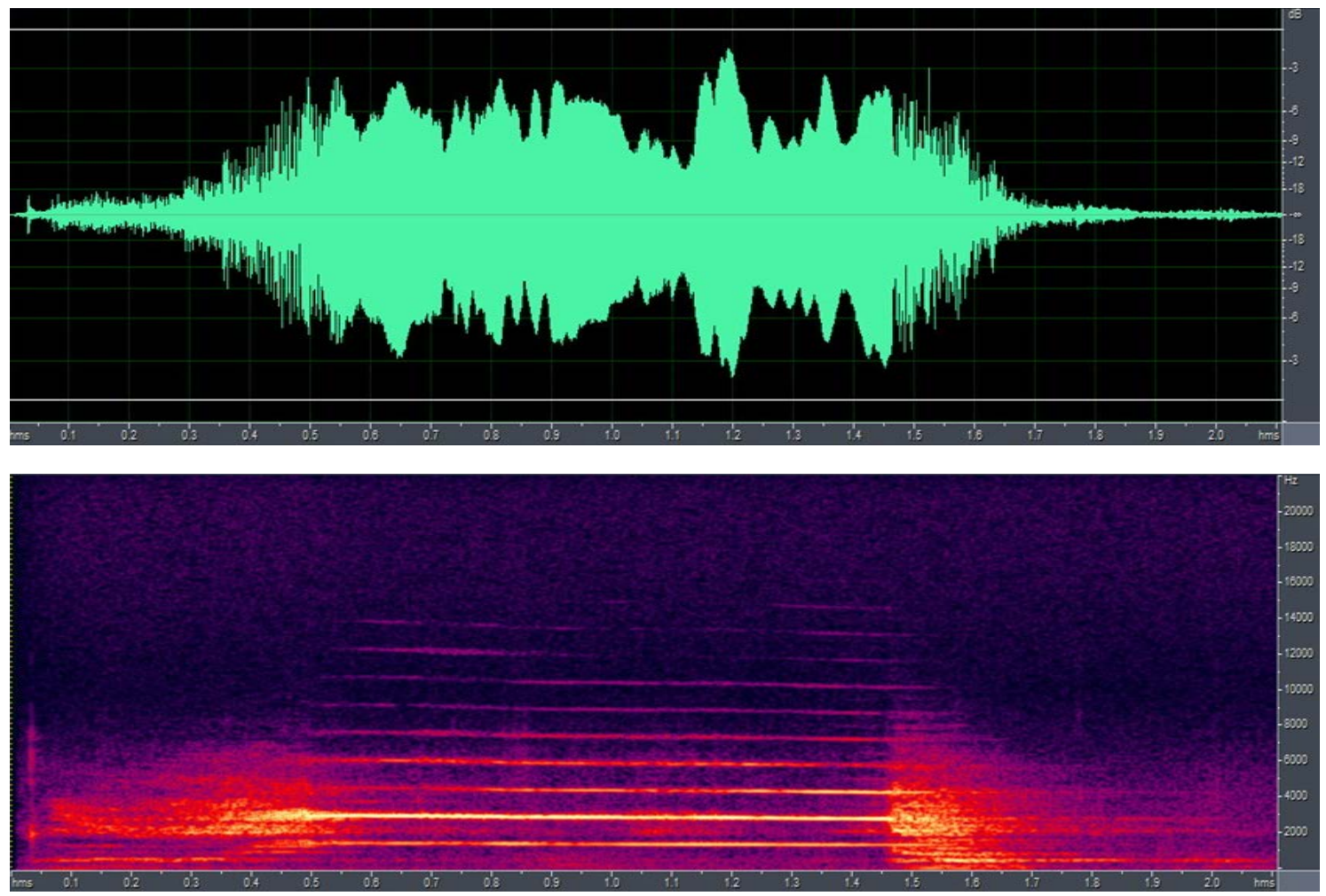

(d)

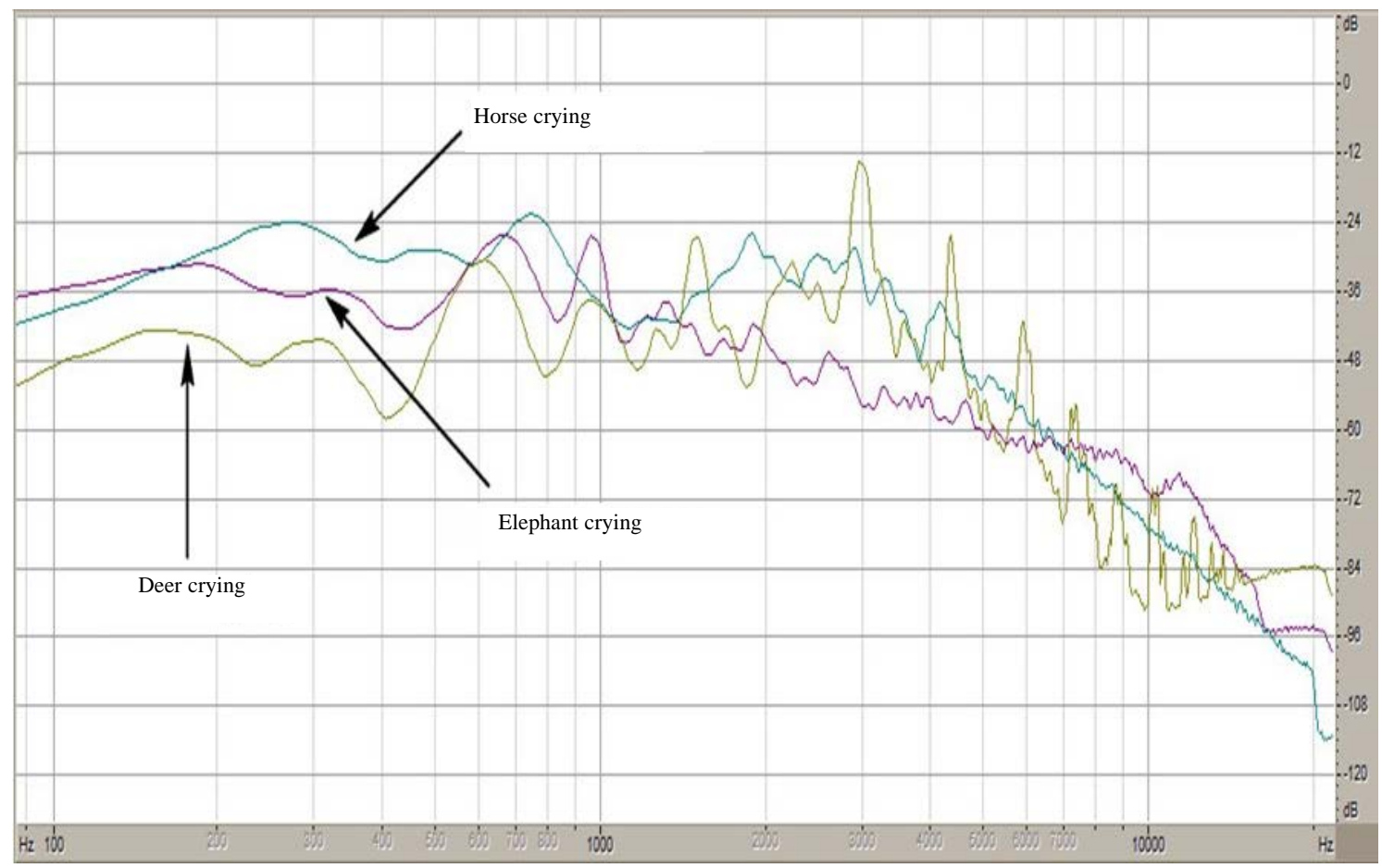

Fig. 2: a) Elephant crying; b) Horse crying; c) Deer crying and d) Herbivorous crying characteristics 




Fig. 3: Comparison of crying frequencies of tigers and elephants

common strong energy. It shows various frequencies even in the high frequency band, constitutes a plurality of pitches and generates a unique high-frequency sound. The elephant cry of a time domain graph shows the characteristics of the cry with the unique rhythm of the elephant which is strong and long. A a of elephant cryo spectrogram shows strong energy even in the frequency band below $1,000 \mathrm{~Hz}$ and it can be seen that the energy is evenly distributed from $1,000 \mathrm{~Hz}$ to the whole band. In the frequency comparison graph of $\mathrm{d}$, the elephant cry has the highest energy in the low frequency band because the elephant is the largest. Because horses and deer are similar kinds of animals, their cries are similar. In the end time domain graph of $\mathrm{b}$ and the deeper time domain graph of $c$, the wide amplitude shows that the energy of the whole frequency band is distributed evenly. The energy spectra of b spectrogram graph and c spectrogram show a slight difference in energy per frequency band but the crying energy characteristics are very similar. A d shows that the frequency pitches of various and unique frequencies are common in the cry of the horse and deer. To compare the cry of carnivorous animals and herbivorous animals, we compared the frequency of the tiger's crying, the representative animal of the predator and the frequency of the elephant crying, a representative animal in the herbivorous animal. The reason for choosing a representative of a predator as a tiger is that the predators are predominantly tigers, lions, leopards and other feline animals and their crying characteristics are similar to each other in their very low frequency characteristics. Among them, the cry of the tiger is the strongest and the sharpest, so, it was chosen as the representative cry of the carnivore. The reason for choosing the herbivorous elephant as an elephant is that herbivores have a variety of cries as well as a wide variety of features. Among them, the elephant has a similar energy of common characteristics of herbivorous animals in the mid-frequency band, this is because it never lags behind the tiger's cry. Herbivorous animals are characterized by high energy in the high frequency region being relatively strong compared to the high frequency energy of the predator while elephant crying is due to the fact that the high frequency sound energy is generally higher than that of the predator, although, it has generally lower energy than other herbivorous animals.

As shown in Fig. 3, the tiger's crying produces not only strong low-frequency sounds below $100 \mathrm{~Hz}$ but also, ultra-low-frequency sounds to strain prey animals. However, the cry of the tiger is lower energy than the strong energy in the low frequency region below $1,000 \mathrm{~Hz}$ and going to the high frequency band. On the contrary, the low frequency energy of the elephant crying $<1,000 \mathrm{~Hz}$ is relatively lower than the tiger crying but it shows a somewhat strong energy. In the mid-frequency and high-frequency regions, the energy levels are generally lower in the herbivorous animals than in the tiger cry, the predator. The reason that herbivorous crying has high energy in the high frequency band is believed to 
be due to the fact that it is necessary to communicate with a large group of colleagues against carnivores. Tigers are almost solitary or if they are young, move to a nuclear family until they grow to some extent. The tiger's crying produces not only strong low-frequency sounds below $100 \mathrm{~Hz}$ but also ultra-low-frequency sounds to strain prey animals. However, the cry of the tiger is lower energy than the strong energy in the low frequency region below $1,000 \mathrm{~Hz}$ and going to the high frequency band. On the contrary, the low frequency energy of the elephant crying $<1,000 \mathrm{~Hz}$ is relatively lower than the tiger crying but it shows a somewhat strong energy. In the mid-frequency and high-frequency regions, the energy levels are generally lower in the herbivorous animals than in the tiger cry, the predator. The reason that herbivorous crying has high energy in the high frequency band is believed to be due to the fact that it is necessary to communicate with a large group of colleagues against carnivores. Tigers are almost solitary or if they are young, move to a nuclear family until they grow to some extent.

It mainly lives in forested forests and alpine areas and eats various animals. The well-developed muscular body and strong jaw with oral structure, the thick neck and the internal organ connected to the belly are harmonized to produce a strong cry and a grand cry. An elephant weighing several tons is the largest animal in the world and one of the largest in the world with 30-40 groups of different family units led by mature females. Among the crowd, there is an elephant who watches when he eats food or when he is asleep and warns him with his voice or footsteps rolling through the ground. The cry of the elephant is uttered by a muscular long nose and a large head bone, a unique oral structure with the highest air cells in the air. Also, the cry of the elephant can be seen to be formed by the influence of the living environment that controls these groups based on these physical conditions.

A study on optimal driving sound of electric power simple transportation system: Carnivores produce strong and rough crying, mainly at low and very low frequency bands. The reason for this is that predators predominantly are felines and they vocalize through a strong skeleton, elastic muscles, a thick neck and a strong vocal cords. The low frequency crying that carnivores produce is used to suppress the prey by ringing the Earth's surface. In fact, most of the herbivorous animals are stuck on the spot when they hear the strong low-frequency sounds of the predators. Particularly, smaller animals are more likely to be unable to move in place because they are more susceptible to the effects of low-frequency noise from carnivorous crying. Herbivorous animals produce strong energy at relatively high frequencies rather than at low frequencies through their large, long, diverse digestive organs, long masses and soft oral structures.

The reason for this high-frequency cry is to surprise predators and send them out to the crowd to alert them that the predators have appeared. Since, herbivores are widely spread and eat grass, it is believed that the cry of the high frequency is made, so that, the cry can be heard and spread widely with a warning sound. The cry of this herbivore is similar to that of a large-sized herbivorous animal but the composition of energy by frequency band is similar. In other words, herbivorous animals have a basic frequency-dependent basic composition ratio and large-sized herbivores are predominantly crying with low and wide spreading and small-sized herbivores are stronger and louder.

\section{CONCLUSION}

Comparisons of carnivorous and herbivorous crying sounds were obtained through this study. It was found that the cries of animals, beyond the means of communication between the same species, contained instinctive functional elements as a means of containment between predators and prey. The distinction between carnivore and herbivorous animals is divided in a large framework according to the type of prey in the food chain ecological environment and there is a conflicting crying characteristic of attack and defense. In other words, it is a characteristic that the cry of the carnivore is used as an aggressive means and that the cry of the herbivore is used as resistance and defensive means. These cries are enough to make each other nervous.

\section{SUGGESTIONS}

We will try to find ways to make use of these animals again for themselves by using the cries of each other to tense each other with the reverse idea. As part of this, the first wild ecological environment can often artificially inflate the cry of each other, making the stagnated natural ecological environment healthier in tension. The second suggests utilization in the zoo. This is because the zoo can revitalize the stagnant environment of the zoo which is inevitable for the zoo. The third suggests that the human protein supplement should be used in an animal feeding environment. Sonar and pigs we are a way to keep our poultry farmers healthy by cultivating a cry of unfamiliar predators. It is the same principle that when you put a catfish into a doodle farm, you will be able to harvest a healthier, more energetic loach. In this way, we will continue to study how to use the sound to improve the ways of benefiting the ecological environment of livestock, livestock and zoo environment. 


\section{REFERENCES}

Bae, M. and M. Kim, 2013. Professor Bae's Sound Story. Gimm-Young Publishers, Seoul, South Korean,.

Bae, S., M. Kim and M. Bae, 2013. On enhancement signal using non-uniform sampling in clipped signals for LTE smart phones. Proceedings of the ICCE Berlin 2013 IEEE 3rd International Conference on Consumer Electronics Berlin (ICCE-Berlin'13), September 9-11, 2013, IEEE, Berlin, Germany, ISBN:978-1-4799-1412-8, pp: $129-130$.

Beckers, G.J.L., B.S. Nelson and R.A. Suthers, 2004. Vocaltract filtering by linguistic articulation in a parrot. Curr. Biol., 14: 1592-1597.

Bum, P., L. Min-Seop and P. Joonhong, 2012. Comparison of sound emission characteristics of diesel engine and gasoline engine sound and artificial engine sound synthesis for sound quality evaluation. Korean Soc. Mech. Eng., 1: 1590-1591.

Chan-Jung, J. and B. Myung-Jin, 2007. A study on the classification of amazed sounds. Acoust. Soc. Korea, 26: $57-58$.

Doo-Hoon, K. and B. Myung-Jin, 2013. Variation of talking elephant's speech characteristics over seven years. Proc. KIIS. Fall Conf., 1: 665-666.
Hoon, S.D. and B.M. Jin, 2010. A study on speaking model of talking elephant. J. Acoust. Soc. Korea Fall Conf., 29: 610-613.

Kyoung, S.Y. and B.M. Jin, 2015. Psychoacoustic study of automobile engine sound. Acoust. Soc. Korea, 34: 180-180.

Lee, D.E., I.G. Hwang, D.I. Jeon and S.S. Park, 2008. Development and optimization of the hybrid engine system model to improve the fuel economy. Trans. Korean Soc. Automot. Eng., 16: 65-73.

Moon, K.B. and Y.Y. Dae, 2015. Design of a green car virtual engine sound generator. Korean Inst. Electr. Eng., 1: 93-94.

Poole, J.H., P.L. Tyack, A.S. Stoeger-Horwath and S. Watwood, 2005. Animal behaviour: Elephants are capable of vocal learning. Nat., 434: 455-456.

Sang-Hwi, J., K. Myung-Sook and B. Myung-Jin, 2017. On designing a new sound of the car-horn. J. Acoust. Soc. Am., 141: 3494-3494.

Se-Bin, J., 2014. A study on the development of safety system for the elderly driver. J. Korea Soc. Automot. Eng., 22: 234-240.

Sung-Hoon, H. and B. Myung-Jin, 2007. A study on the sound that enhances concentration. IEIC. Conf. Fall, 30: $671-672$. 